\title{
Factorization by Invariant Embedding of a Boundary Value Problem for the Laplace Operator
}

\author{
Jacques Henry ${ }^{1}$, Bento Louro ${ }^{2}$, and Maria C. Soares ${ }^{2}$ \\ 1 INRIA Bordeaux Sud-Ouest, IMB Université Bordeaux 1 351, cours de la libération, \\ 33405 Talence, France \\ jacques.henry@inria.fr \\ 2 Departamento de Matemática, Faculdade de Ciências e Tecnologia, \\ Universidade Nova de Lisboa, Monte de Caparica, 2829-516 Caparica, Portugal \\ bjlafct.unl.pt, mcs@fct.unl.pt
}

\begin{abstract}
This work concerns the factorization of a second order elliptic boundary value problem defined in a star-shaped bounded regular domain, in a system of uncoupled first order initial value problems, using the technique of invariant embedding. The family of domains is defined by a homothety. The method yields an equivalent formulation to the initial boundary value problem by a system of two uncoupled Cauchy problems. The singularity at the origin of the homothety is studied.
\end{abstract}

\section{Introduction}

The invariant embedding technique consists in embedding the initial problem in a family of similar problems depending on a parameter, which are solved recursively. It has been used by Bellman [1] and Lions [6] (in the infinite dimensional case) to derive the optimal feedback law in linear-quadratic optimal control problems. It yields a factorization of the optimality system. In our approach, the invariant embedding is used spatially. Each problem is defined over a subdomain limited by a mobile boundary (see Fig. 1, depending on the parameter. Defining an operator relating the value of the solution, or its derivative, with the mobile boundary condition (Dirichlet-Neumann or NeumannDirichlet, for example), we find a family of operators on functions defined on the mobile boundary satisfying a Riccati equation. The method applied to cylindrical domains has been presented in [4]. Here we particularize the study to the two dimensional Poisson equation with a Dirichlet boundary condition: $-\Delta u=f$, in $\Omega, u_{\left.\right|_{\Gamma_{a}}}=0$. The assumption on the shape of the domain is less restrictive than in [4]5] and the invariant embedding is realized by a homothety. The family of curves which limits the subdomains defined by the invariant embedding are homothetic to one another, and we consider the moving boundary starting on the outside boundary of the domain and shrinking to a point. We show some results dealing with the singularity that will appear at that point. The factorization of the boundary value problem can be viewed as an infinite dimensional generalization of the block Gauss $L U$ factorization.

A. Korytowski et al. (Eds.): System Modeling and Optimization, IFIP AICT 312, pp. 282 292, 2009.

(c) IFIP International Federation for Information Processing 2009 


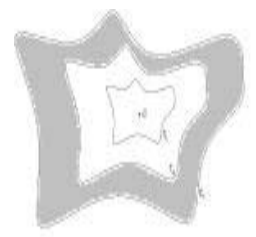

Fig. 1. The star-shaped domain

\section{Definition of the Problem and Regularization}

Let $\Omega \subset \mathbb{R}^{2}$ be an open bounded regular domain containing the origin $\mathrm{O}$, star-shaped with respect to $\mathrm{O}$, with boundary $\Gamma_{a}=\partial \Omega$. We consider the Poisson problem with Dirichlet data

$$
(\mathscr{P})\left\{\begin{array}{l}
-\Delta u=f, \text { in } \Omega \\
\left.u\right|_{\Gamma_{a}}=0,
\end{array}\right.
$$

where $f \in L^{2}(\Omega)$. In spite of the particularization to the Laplacian operator in this definition, we believe that the same procedure could be applied to any strongly elliptic self-adjoint problem.

Applying the (spatial) invariant embedding method to this problem, we must start defining a family of subdomains limited by a boundary sweeping over the initial domain $\Omega$.

We start dealing with the case where the family of curves which limits the subdomains, starts on the boundary of the domain and shrinks homothetically to a point. Since the mobile boundary reduces to a point, a singularity will necessary appear at that point. We must make, as a consequence, a regularization around this point and a possible way to do it, is to define an auxiliary domain, where we introduce a fictitious boundary around the singular point. In this case, however, we introduce a perturbation of the solution so, naturally, we must choose the new boundary condition, in a way that we can obtain the convergence of this auxiliary problem to the initial one. With this purpose, we will consider the following auxiliary problem:

$$
\left(\mathscr{P}_{\varepsilon}\right)\left\{\begin{array}{l}
-\Delta u_{\varepsilon}=f, \text { in } \Omega \backslash \Omega_{\varepsilon} \\
\left.u_{\varepsilon}\right|_{\Gamma_{a}}=0 \\
\int_{\Gamma_{\varepsilon}} \frac{\partial u_{\varepsilon}}{\partial n} \mathrm{~d} \Gamma=0,\left.u_{\varepsilon}\right|_{\Gamma_{\varepsilon}} \text { is constant. }
\end{array}\right.
$$

Here, $\Omega_{\varepsilon}$ is an open regular domain verifying $\bar{\Omega}_{\varepsilon} \subset \Omega$ and $\Gamma_{\varepsilon}$, which is homothetical to $\Gamma_{a}$ with ratio $\varepsilon<1$, is the boundary of $\Omega_{\varepsilon}$. This problem is well posed. We can justify the choice of the boundary conditions on $\Gamma_{\varepsilon}$ with the fact that the condition

$$
\int_{\Gamma_{\varepsilon}} \frac{\partial u_{\varepsilon}}{\partial n} \mathrm{~d} \Gamma_{\varepsilon}=0
$$

corresponds to a null total flux. 
It's easy to see that $U_{\varepsilon}=\left\{u_{\varepsilon} \in H^{1}\left(\Omega \backslash \Omega_{\varepsilon}\right): u_{\varepsilon_{\left.\right|_{a}}}=0 \wedge u_{\varepsilon_{\Gamma_{\varepsilon}}}\right.$ is constant $\}$ is a Hilbert space and that the variational formulation of problem $\left(\mathscr{P}_{\varepsilon}\right)$ is

$$
\left\{\begin{array}{l}
u_{\varepsilon} \in U_{\varepsilon} \\
\int_{\Omega \backslash \Omega_{\varepsilon}} \nabla u_{\varepsilon} \nabla v_{\varepsilon} \mathrm{d} \Omega=\int_{\Omega \backslash \Omega_{\varepsilon}} f v_{\varepsilon} \mathrm{d} \Omega, \forall v_{\varepsilon} \in U_{\varepsilon} .
\end{array}\right.
$$

We prove that when $\varepsilon \rightarrow 0$, problem $\left(\mathscr{P}_{\varepsilon}\right)$ reduces to problem $(\mathscr{P})$, that is, $u_{\varepsilon}$, the solution of problem $\left(\mathscr{P}_{\varepsilon}\right)$, converges to $u$, the solution of problem $(\mathscr{P})$, by means of the next theorem, which proof can be found in [3]:

Theorem 1. Let $u_{\varepsilon}$ (respectively, $\left.u\right)$ be the solution of $\left(\mathscr{P}_{\varepsilon}\right)($ respectively, $(\mathscr{P}))$ and

$$
\tilde{u}_{\varepsilon}=\left\{\begin{array}{l}
u_{\varepsilon}, \text { in } \Omega \backslash \Omega_{\varepsilon} \\
u_{\varepsilon}=\left.u_{\varepsilon}\right|_{\Gamma_{\varepsilon}}, \text { in } \Omega_{\varepsilon} .
\end{array}\right.
$$

Then,

$$
\tilde{u}_{\varepsilon} \underset{\varepsilon \rightarrow 0}{\rightarrow} u, H^{1}(\Omega)-\text { strong. }
$$

\section{Invariant Embedding in a Star-Shaped Domain}

We start defining polar coordinates by means of $x=\rho \cos (\theta), y=\rho \sin (\theta), 0<\rho \leq$ $\varphi(\theta)$, where $\rho=\varphi(\theta)$ defines the boundary $\Gamma_{a}$. Here, $\varphi(\theta) \in \mathscr{C}^{1}([0,2 \pi])$ is such that $\varphi(2 \pi)=\varphi(0), \varphi^{\prime}(2 \pi)=\varphi^{\prime}(0)$ and $0<k_{0}<\varphi(\theta)<k_{1}$. In the coordinates $(\tau, \theta)$, where $\tau=\rho / \varphi(\theta)$, the Laplace equation becomes

$$
\begin{aligned}
& \left(\frac{1}{\varphi^{2}(\theta)}+\frac{\left(\varphi^{\prime}(\theta)\right)^{2}}{\varphi^{4}(\theta)}\right) \frac{\partial^{2} u}{\partial \tau^{2}}+\left(-2 \frac{\varphi^{\prime}(\theta)}{\varphi^{3}(\theta)}\right) \frac{1}{\tau} \frac{\partial^{2} u}{\partial \tau \partial \theta} \\
& +\left(-\frac{\varphi^{\prime \prime}(\theta)}{\varphi^{3}(\theta)}+2 \frac{\left(\varphi^{\prime}(\theta)\right)^{2}}{\varphi^{4}(\theta)}+\frac{1}{\varphi^{2}(\theta)}\right) \frac{1}{\tau} \frac{\partial u}{\partial \tau}+\frac{1}{\varphi^{2}(\theta)} \frac{1}{\tau^{2}} \frac{\partial^{2} u}{\partial \theta^{2}}=-f
\end{aligned}
$$

Now, let $\alpha$ be the angle $(\mathbf{O M}, \mathbf{n})$ where $M$ is a point on $\Gamma_{a}$ and $\mathbf{n}$ is the outward normal to $\Gamma_{a}$ at $M$. We assume that $-\pi / 2<\alpha_{0} \leq \alpha \leq \alpha_{1}<\pi / 2$. We consider the homothety of center $O$ and ratio $0<\tau<1$, which transforms $\Omega$ to $\Omega_{\tau}$ with boundary $\Gamma_{\tau}$, and the following system of curvilinear coordinates: for $M \in \Omega,(\tau, t)$ are such that $M^{\prime}$, the image of $M$ by a $1 / \tau$ homothety, belongs to $\Gamma_{a}$ and $t, 0 \leq t<t_{0}$, is the curvilinear abscissa of $M^{\prime}$ on $\Gamma_{a}$ (where $t_{0}$ is the length of $\Gamma_{a}$ ). This new system of coordinates and the one defined previously are related through the equalities $\cos (\alpha) \mathrm{d} t=\varphi \mathrm{d} \theta$ and $\tan (\alpha)=\frac{\varphi^{\prime}}{\varphi}$. In these coordinates, the exterior normal to $\Gamma_{\tau}$ can be written as $\frac{\partial}{\partial n}=$ $-\frac{1}{\varphi \cos (\alpha)} \frac{\partial}{\partial \tau}+\frac{\tan (\alpha)}{\tau} \frac{\partial}{\partial t}$, and the Laplace equation takes the form

$$
\begin{aligned}
& -\frac{\partial}{\partial \tau}\left(\frac{\tau}{\varphi \cos (\alpha)} \frac{\partial u_{\tau}}{\partial \tau}-\tan (\alpha) \frac{\partial u_{\tau}}{\partial t}\right) \\
& -\frac{\partial}{\partial t}\left(-\tan (\alpha) \frac{\partial u_{\tau}}{\partial \tau}+\frac{\varphi}{\tau \cos (\alpha)} \frac{\partial u_{\tau}}{\partial t}\right)=\tau f \varphi \cos (\alpha) .
\end{aligned}
$$


Using the technique of invariant embedding, we embed problem $\left(\mathscr{P}_{\varepsilon}\right)$ in a family of similar problems defined on $\Omega \backslash \Omega_{\tau}=\{(s, t) \in] \tau, 1[\times] 0, t_{0}[\}$, for every $\tau \in[\varepsilon, 1)$. For each problem we impose the Neumann boundary condition $\left.\frac{\partial u_{\tau}}{\partial n}\right|_{\Gamma_{\tau}}=h$, where $\Gamma_{\tau}$ is the moving boundary:

$$
\left(\mathscr{P}_{\tau, h}\right)\left\{\begin{array}{l}
-\frac{\partial}{\partial \tau}\left(\frac{\tau}{\varphi \cos (\alpha)} \frac{\partial u_{\tau}}{\partial \tau}-\tan (\alpha) \frac{\partial u_{\tau}}{\partial t}\right) \\
-\frac{\partial}{\partial t}\left(-\tan (\alpha) \frac{\partial u_{\tau}}{\partial \tau}+\frac{\varphi}{\tau \cos (\alpha)} \frac{\partial u_{\tau}}{\partial t}\right)=\tau f \varphi \cos (\alpha), \text { in } \Omega \backslash \Omega_{\tau} \\
\left.u_{\tau}\right|_{\Gamma_{a}}=0 \\
u_{\tau_{t=0}}=u_{\tau_{t=t_{0}}},\left.\frac{\partial u_{\tau}}{\partial t}\right|_{t=0}=\left.\frac{\partial u_{\tau}}{\partial t}\right|_{t=t_{0}} \\
\left.\frac{\partial u_{\tau}}{\partial n}\right|_{\Gamma_{\tau}}=-\frac{1}{\varphi \cos (\alpha)} \frac{\partial u_{\tau}}{\partial \tau}+\frac{\tan (\alpha)}{\tau} \frac{\partial u_{\tau}}{\partial t}=h .
\end{array}\right.
$$

In order to apply a method similar to the one used by Lions [6] for decoupling the optimality conditions associated to an optimal control problem of a parabolic equation, we define $P(\tau) h=\gamma_{\tau_{\Gamma_{\tau}}}$, where $\gamma_{\tau}$ is the solution of

$$
\left\{\begin{array}{l}
\frac{\partial}{\partial \tau}\left(\frac{\tau}{\varphi \cos (\alpha)} \frac{\partial \gamma_{\tau}}{\partial \tau}-\tan (\alpha) \frac{\partial \gamma_{\tau}}{\partial t}\right) \\
+\frac{\partial}{\partial t}\left(-\tan (\alpha) \frac{\partial \gamma_{\tau}}{\partial \tau}+\frac{\varphi}{\tau \cos (\alpha)} \frac{\partial \gamma_{\tau}}{\partial t}\right)=0, \text { in } \Omega \backslash \Omega_{\tau} \\
\gamma_{\tau_{\Gamma_{a}}}=0 \\
\left.\frac{\partial \gamma_{\tau}}{\partial n}\right|_{\Gamma_{\tau}}=h \\
\gamma_{\tau_{t=0}}=\gamma_{\tau_{t=t_{0}}},\left.\frac{\partial \gamma_{\tau}}{\partial t}\right|_{t=0}=\left.\frac{\partial \gamma_{\tau}}{\partial t}\right|_{t=t_{0}}
\end{array}\right.
$$

and $r(\tau)=\beta_{\tau_{\Gamma_{\tau}}}$, where $\beta_{\tau}$ is the solution of

$$
\left\{\begin{array}{l}
-\frac{\partial}{\partial \tau}\left(\frac{\tau}{\varphi \cos (\alpha)} \frac{\partial \beta_{\tau}}{\partial \tau}-\tan (\alpha) \frac{\partial \beta_{\tau}}{\partial t}\right) \\
-\frac{\partial}{\partial t}\left(-\tan (\alpha) \frac{\partial \beta_{\tau}}{\partial \tau}+\frac{\varphi}{\tau \cos (\alpha)} \frac{\partial \beta_{\tau}}{\partial t}\right)=\tau f \varphi \cos (\alpha), \text { in } \Omega \backslash \Omega_{\tau} \\
\beta_{\tau_{\Gamma_{a}}}=0 \\
\left.\frac{\partial \beta_{\tau}}{\partial n}\right|_{\Gamma_{\tau}}=0 \\
\beta_{\tau_{t=0}}=\beta_{\tau_{\mid t=t_{0}}},\left.\frac{\partial \beta_{\tau}}{\partial t}\right|_{t=0}=\left.\frac{\partial \beta_{\tau}}{\partial t}\right|_{t=t_{0}} .
\end{array}\right.
$$


By linearity of $\left(\mathscr{P}_{\tau, h}\right)$, the following relation holds true

$$
u_{\tau}(\tau)=P(\tau) h+r(\tau)
$$

Then taking $h=\frac{\partial u}{\partial n}(\tau)$ on $\Gamma_{\tau}$, it is clear that $u_{\tau}\left(\tau^{\prime}\right)=u\left(\tau^{\prime}\right)$ for $\tau \leq \tau^{\prime} \leq 1$. Then

$$
u(\tau)=P(\tau) \frac{\partial u}{\partial n}(\tau)+r(\tau)
$$

Taking the derivative of the previous equality, in a formal way, with respect to $\tau$, we can derive (cf Sect.4) the following system of uncoupled, first order in $\tau$, equations:

$$
\left\{\begin{array}{l}
\frac{\partial P}{\partial \tau}-\frac{\varphi \sin (\alpha)}{\tau} \frac{\partial}{\partial t} P+\frac{P}{\tau} \frac{\partial}{\partial t}(\varphi \sin (\alpha))+\frac{P}{\tau^{2}} \frac{\partial}{\partial t}\left(\varphi \cos (\alpha) \frac{\partial}{\partial t} P\right) \\
-\frac{P}{\tau}=-\varphi \cos (\alpha) \mathrm{I} \\
\frac{\partial r}{\partial \tau}-\frac{\varphi \sin (\alpha)}{\tau} \frac{\partial r}{\partial t}+\frac{P}{\tau^{2}} \frac{\partial}{\partial t}\left(\varphi \cos (\alpha) \frac{\partial r}{\partial t}\right)=-P f \varphi \cos (\alpha) \\
P\left(-\frac{1}{\varphi \cos (\alpha)} \frac{\partial u}{\partial \tau}+\frac{\tan (\alpha)}{\tau} \frac{\partial u}{\partial t}\right)+r=u .
\end{array}\right.
$$

Again from (13), and considering the initial conditions on $\Gamma_{a}$, we also obtain $P(1)=0$ and $r(1)=0$, which corresponds to the initial conditions of the first two equations above. We will define the initial condition for the last equation in Sect. 5] The Riccati equation for $P$ which depends only on the operator and the shape of the domain in problem $\mathscr{P}$, can be solved for decreasing $\tau$ once for all. For each data $(f)$, the problem is now solved by integrating two Cauchy problems: the one on $r$ for $\tau$ decreasing from 1 to 0 and the one on $u$ backwards in $\tau$.

\section{Arriving to the Uncoupled System}

Considering $u_{\varepsilon}$, solution of (4) and $v_{\varepsilon}$, solution of the homogeneous equation $\Delta v_{\varepsilon}=0$ with arbitrary boundary condition on $\Gamma_{\varepsilon}$ and using the Green formula, we obtain in the $(\tau, t)$ coordinates

$$
\begin{aligned}
& \int_{\varepsilon}^{1} \int_{0}^{t_{0}} \frac{\tau}{\varphi \cos (\alpha)} \frac{\partial v_{\varepsilon}}{\partial \tau} \frac{\partial u_{\varepsilon}}{\partial \tau}-\tan (\alpha)\left(\frac{\partial v_{\varepsilon}}{\partial t} \frac{\partial u_{\varepsilon}}{\partial \tau}+\frac{\partial v_{\varepsilon}}{\partial \tau} \frac{\partial u_{\varepsilon}}{\partial t}\right) \\
& +\frac{\varphi}{\tau \cos (\alpha)} \frac{\partial v_{\varepsilon}}{\partial t} \frac{\partial u_{\varepsilon}}{\partial t} \mathrm{~d} t \mathrm{~d} \tau=-\left.\left.\varepsilon \int_{0}^{t_{0}}\left(\frac{1}{\varphi \cos (\alpha)} \frac{\partial v_{\varepsilon}}{\partial \tau}-\frac{\tan (\alpha)}{\varepsilon} \frac{\partial v_{\varepsilon}}{\partial t}\right)\right|_{\tau=\varepsilon} u_{\varepsilon}\right|_{\tau=\varepsilon} \mathrm{d} t
\end{aligned}
$$

A similar formula holds for $\Omega \backslash \Omega_{\tau}=\{(s, t) \in] \tau, 1[\times] 0, t_{0}[\}$. 
Deriving the resulting equality with respect to the variable $\tau$, we obtain

$$
\begin{aligned}
& \frac{\partial}{\partial \tau}\left(\int _ { \tau } ^ { 1 } \int _ { 0 } ^ { t _ { 0 } } \left(\frac{s}{\varphi \cos (\alpha)} \frac{\partial v_{\tau}}{\partial \tau} \frac{\partial u_{\tau}}{\partial \tau}-\tan (\alpha)\left(\frac{\partial v_{\tau}}{\partial t} \frac{\partial u_{\tau}}{\partial \tau}+\frac{\partial v_{\tau}}{\partial \tau} \frac{\partial u_{\tau}}{\partial t}\right)\right.\right. \\
& \left.\left.+\frac{\varphi}{s \cos (\alpha)} \frac{\partial v_{\tau}}{\partial t} \frac{\partial u_{\tau}}{\partial t}\right) \mathrm{~d} t \mathrm{~d} s\right)=-\frac{\partial}{\partial \tau}\left(\int_{0}^{t_{0}}\left(\frac{\tau}{\varphi \cos (\alpha)} \frac{\partial v_{\tau}}{\partial \tau}-\tan (\alpha) \frac{\partial v_{\tau}}{\partial t}\right) u_{\tau} \mathrm{d} t\right) \Rightarrow \\
& \int_{0}^{t_{0}}\left(\frac{\tau}{\varphi \cos (\alpha)} \frac{\partial v_{\tau}}{\partial \tau} \frac{\partial u_{\tau}}{\partial \tau}-\tan (\alpha)\left(\frac{\partial v_{\tau}}{\partial t} \frac{\partial u_{\tau}}{\partial \tau}+\frac{\partial v_{\tau}}{\partial \tau} \frac{\partial u_{\tau}}{\partial t}\right)+\frac{\varphi}{\tau \cos (\alpha)} \frac{\partial v_{\tau}}{\partial t} \frac{\partial u_{\tau}}{\partial t}\right) \mathrm{d} t \\
& =\int_{0}^{t_{0}} \frac{\partial}{\partial \tau}\left(\left(\frac{\tau}{\varphi \cos (\alpha)} \frac{\partial v_{\tau}}{\partial \tau}-\tan (\alpha) \frac{\partial v_{\tau}}{\partial t}\right) u_{\tau}\right) \mathrm{d} t=-\int_{0}^{t_{0}} \frac{\partial}{\partial \tau}\left(\tau \frac{\partial v_{\tau}}{\partial n} u_{\tau}\right) \mathrm{d} t .
\end{aligned}
$$

Then, using (13) and the Laplace equation (8) we have successively,

$$
\begin{aligned}
\int_{0}^{t_{0}} & \left(\frac{\tau}{\varphi \cos (\alpha)} \frac{\partial v_{\tau}}{\partial \tau} \frac{\partial u_{\tau}}{\partial \tau}-\tan (\alpha)\left(\frac{\partial v_{\tau}}{\partial t} \frac{\partial u_{\tau}}{\partial \tau}+\frac{\partial v_{\tau}}{\partial \tau} \frac{\partial u_{\tau}}{\partial t}\right)+\frac{\varphi}{\tau \cos (\alpha)} \frac{\partial v_{\tau}}{\partial t} \frac{\partial u_{\tau}}{\partial t}\right) \mathrm{d} t \\
= & -\int_{0}^{t_{0}}\left(\frac{\partial}{\partial \tau}\left(\tau \frac{\partial v_{\tau}}{\partial n}\right) P \frac{\partial u_{\tau}}{\partial n}+\tau \frac{\partial v_{\tau}}{\partial n} \frac{\partial P}{\partial \tau} \frac{\partial u_{\tau}}{\partial n}+\tau \frac{\partial v_{\tau}}{\partial n} P \frac{\partial}{\partial \tau}\left(\frac{\partial u_{\tau}}{\partial n}\right)\right. \\
& \left.+\frac{\partial}{\partial \tau}\left(\tau \frac{\partial v_{\tau}}{\partial n}\right) r+\tau \frac{\partial v_{\tau}}{\partial n} \frac{\partial r}{\partial \tau}\right) \mathrm{d} t \\
= & \int_{0}^{t_{0}}\left(\frac{\partial}{\partial t}\left(\tan (\alpha) \frac{\partial v_{\tau}}{\partial \tau}-\frac{\varphi}{\tau \cos (\alpha)} \frac{\partial v_{\tau}}{\partial t}\right) P \frac{\partial u_{\tau}}{\partial n}-\tau \frac{\partial v_{\tau}}{\partial n} \frac{\partial P}{\partial \tau} \frac{\partial u_{\tau}}{\partial n}+\frac{\partial v_{\tau}}{\partial n} P \frac{\partial u_{\tau}}{\partial n}\right. \\
& +\frac{\partial v_{\tau}}{\partial n} P\left[\frac{\partial}{\partial t}\left(\tan (\alpha) \frac{\partial u_{\tau}}{\partial \tau}-\frac{\varphi}{\tau \cos (\alpha)} \frac{\partial u_{\tau}}{\partial t}\right)-f \tau \varphi \cos (\alpha)\right] \\
& \left.+\frac{\partial}{\partial t}\left(\tan (\alpha) \frac{\partial v_{\tau}}{\partial \tau}-\frac{\varphi}{\tau \cos (\alpha)} \frac{\partial v_{\tau}}{\partial t}\right) r-\tau \frac{\partial v_{\tau}}{\partial n} \frac{\partial r}{\partial \tau}\right) \mathrm{d} t
\end{aligned}
$$

Therefore, using once again (13) and integrating by parts in $t$ the right hand side, the equality becomes

$$
\begin{aligned}
& \int_{0}^{t_{0}}\left(\frac{\tau}{\varphi \cos (\alpha)} \frac{\partial v_{\tau}}{\partial \tau} \frac{\partial u_{\tau}}{\partial \tau}-\tan (\alpha) \frac{\partial v_{\tau}}{\partial t} \frac{\partial u_{\tau}}{\partial \tau}-\tan (\alpha) \frac{\partial v_{\tau}}{\partial \tau} \frac{\partial u_{\tau}}{\partial t}\right. \\
& \left.\quad+\frac{\varphi}{\tau \cos (\alpha)} \frac{\partial v_{\tau}}{\partial t} \frac{\partial u_{\tau}}{\partial t}\right) \mathrm{d} t \\
& =\int_{0}^{t_{0}}\left(-\tan (\alpha) \frac{\partial v_{\tau}}{\partial \tau} \frac{\partial u_{\tau}}{\partial t}+\frac{\varphi}{\tau \cos (\alpha)} \frac{\partial v_{\tau}}{\partial t} \frac{\partial u_{\tau}}{\partial t}\right. \\
& -\tau \frac{\partial v_{\tau}}{\partial n} \frac{\partial P}{\partial \tau} \frac{\partial u_{\tau}}{\partial n}+\frac{\partial v_{\tau}}{\partial n} P \frac{\partial u_{\tau}}{\partial n}-\frac{\partial v_{\tau}}{\partial t} \tan (\alpha) \frac{\partial u_{\tau}}{\partial \tau}+\frac{\partial v_{\tau}}{\partial t} \frac{\varphi}{\tau \cos (\alpha)} \frac{\partial u_{\tau}}{\partial t} \\
& \left.\quad-\frac{\partial v_{\tau}}{\partial n} P f \tau \varphi \cos (\alpha)-\tau \frac{\partial v_{\tau}}{\partial n} \frac{\partial r}{\partial \tau}\right) \mathrm{d} t .
\end{aligned}
$$


After simplification, we obtain

$$
\begin{aligned}
& \int_{0}^{t_{0}} \frac{\tau}{\varphi \cos (\alpha)} \frac{\partial v_{\tau}}{\partial \tau} \frac{\partial u_{\tau}}{\partial \tau} \mathrm{d} t \\
& =\int_{0}^{t_{0}}\left(-\tau \frac{\partial v_{\tau}}{\partial n} \frac{\partial P}{\partial \tau} \frac{\partial u_{\tau}}{\partial n}+\frac{\partial v_{\tau}}{\partial n} P \frac{\partial u_{\tau}}{\partial n}+\frac{\partial v_{\tau}}{\partial t} \frac{\varphi}{\tau \cos (\alpha)} \frac{\partial u_{\tau}}{\partial t}\right. \\
& \left.\quad-\frac{\partial v_{\tau}}{\partial n} P f \tau \varphi \cos (\alpha)-\tau \frac{\partial v_{\tau}}{\partial n} \frac{\partial r}{\partial \tau}\right) \mathrm{d} t .
\end{aligned}
$$

Expressing the $t$ and $\tau$ derivatives in terms of normal derivatives, we get

$$
\begin{aligned}
\int_{0}^{t_{0}} & \left(\tau \varphi \cos (\alpha) \frac{\partial v_{\tau}}{\partial n} \frac{\partial u_{\tau}}{\partial n}-\varphi \sin (\alpha) \frac{\partial v_{\tau}}{\partial n} \frac{\partial}{\partial t}\left(P \frac{\partial u_{\tau}}{\partial n}\right)\right. \\
& \left.-\varphi \sin (\alpha) \frac{\partial v_{\tau}}{\partial n} \frac{\partial r}{\partial t}-\varphi \sin (\alpha) \frac{\partial}{\partial t}\left(P \frac{\partial v_{\tau}}{\partial n}\right) \frac{\partial u_{\tau}}{\partial n}\right) \mathrm{d} t \\
& =\int_{0}^{t_{0}}\left(-\tau \frac{\partial v_{\tau}}{\partial n} \frac{\partial P}{\partial \tau} \frac{\partial u_{\tau}}{\partial n}+\frac{\partial v_{\tau}}{\partial n} P \frac{\partial u_{\tau}}{\partial n}-\tau \frac{\partial v_{\tau}}{\partial n} \frac{\partial r}{\partial \tau}\right. \\
& +\frac{\varphi \cos (\alpha)}{\tau} \frac{\partial}{\partial t}\left(P \frac{\partial v_{\tau}}{\partial n}\right) \frac{\partial}{\partial t}\left(P \frac{\partial u_{\tau}}{\partial n}\right) \\
& \left.+\frac{\varphi \cos (\alpha)}{\tau} \frac{\partial}{\partial t}\left(P \frac{\partial v_{\tau}}{\partial n}\right) \frac{\partial r}{\partial t}-\frac{\partial v_{\tau}}{\partial n} P f \tau \varphi \cos (\alpha)\right) \mathrm{d} t
\end{aligned}
$$

From the principle of invariant embedding, $\frac{\partial u_{\tau}}{\partial n}$ on $\Gamma_{\tau}$ is arbitrary so that we can separate the parts depending and independent of this quantity, obtaining

$$
\begin{aligned}
& \left(\tau \varphi \cos (\alpha) \frac{\partial v_{\tau}}{\partial n}, \frac{\partial u_{\tau}}{\partial n}\right)-\left(\varphi \sin (\alpha) \frac{\partial}{\partial t} \circ P \frac{\partial v_{\tau}}{\partial n}, \frac{\partial u_{\tau}}{\partial n}\right) \\
& -\left(\varphi \sin (\alpha) \frac{\partial v_{\tau}}{\partial n}, \frac{\partial}{\partial t} \circ P \frac{\partial u_{\tau}}{\partial n}\right)=\left(-\tau \frac{\partial v_{\tau}}{\partial n}, \frac{\partial P}{\partial \tau} \frac{\partial u_{\tau}}{\partial n}\right) \\
& +\left(\frac{\partial v_{\tau}}{\partial n}, P \frac{\partial u_{\tau}}{\partial n}\right)+\left(\frac{\varphi \cos (\alpha)}{\tau} \frac{\partial}{\partial t} \circ P \frac{\partial v_{\tau}}{\partial n}, \frac{\partial}{\partial t} \circ P \frac{\partial u_{\tau}}{\partial n}\right)
\end{aligned}
$$

and

$$
\begin{aligned}
& \left(\varphi \sin (\alpha) \frac{\partial v_{\tau}}{\partial n}, \frac{\partial r}{\partial t}\right)=\left(P \frac{\partial v_{\tau}}{\partial n}, f \tau \varphi \cos (\alpha)\right)+\left(\tau \frac{\partial v_{\tau}}{\partial n}, \frac{\partial r}{\partial \tau}\right) \\
& -\left(\frac{\varphi \cos (\alpha)}{\tau} \frac{\partial}{\partial t} \circ P \frac{\partial v_{\tau}}{\partial n}, \frac{\partial r}{\partial t}\right),
\end{aligned}
$$

where $\frac{\partial v_{\tau}}{\partial n}$ is an arbitrary test function. This corresponds to 14 .

\section{Defining $u(0)$}

In this section we study the limit of problem $\left(\mathscr{P}_{\varepsilon}\right)$ when $\varepsilon$ goes to zero, that is when the hole shrinks to the origin. This is useful to define an initial condition for the equation for $u$ in the factorized form. 
Theorem 2. Considering $u_{\varepsilon}$ the solution of problem $\left(\mathscr{P}_{\varepsilon}\right),\left.u_{\varepsilon}\right|_{\Gamma_{\varepsilon}}$ is bounded by a constant not depending on $\varepsilon$.

Proof. The first part of the proof consists on showing that we have

$$
\inf _{\Gamma_{\varepsilon}} w_{\varepsilon} \leq\left. u_{\varepsilon}\right|_{\Gamma_{\varepsilon}} \leq \sup _{\Gamma_{\varepsilon}} w_{\varepsilon}
$$

where $w_{\varepsilon} \in H_{0}^{1}(\Omega)$ is the solution of the problem

$$
-\Delta w_{\varepsilon}=\tilde{f}_{\varepsilon}=\left\{\begin{array}{l}
f, \Omega \backslash \Omega_{\varepsilon} \\
0, \Omega_{\varepsilon} .
\end{array}\right.
$$

From $-\Delta w_{\varepsilon}=\tilde{f}_{\varepsilon}$, in $H_{0}^{1}(\Omega)$, we find

$$
\int_{\Omega}-\Delta w_{\varepsilon}=\int_{\Omega} \tilde{f}_{\varepsilon}=\int_{\Omega \backslash \Omega_{\varepsilon}} f=-\int_{\Gamma_{a}} \frac{\partial w_{\varepsilon}}{\partial n} .
$$

On the other hand, from the formulation of problem $\left(\mathscr{P}_{\varepsilon}\right)$ and choosing a test function equal to one, we find

$$
\int_{\Omega \backslash \Omega_{\varepsilon}}-\Delta u_{\varepsilon}=\int_{\Omega \backslash \Omega_{\varepsilon}} f=-\int_{\Gamma_{\varepsilon}} \frac{\partial u_{\varepsilon}}{\partial n}-\int_{\Gamma_{a}} \frac{\partial u_{\varepsilon}}{\partial n}=-\int_{\Gamma_{a}} \frac{\partial u_{\varepsilon}}{\partial n} .
$$

Therefore, we have the equality $\int_{\Gamma_{a}} \frac{\partial u_{\varepsilon}}{\partial n}=\int_{\Gamma_{a}} \frac{\partial w_{\varepsilon}}{\partial n}$.

Let us now suppose that $\left.u_{\varepsilon}\right|_{\Gamma_{\varepsilon}}=c_{\varepsilon}<\inf _{\Gamma_{\varepsilon}} w_{\varepsilon}$. Then, $u_{\varepsilon}-w_{\varepsilon}$ satisfies:

$$
\left\{\begin{array}{l}
-\Delta\left(u_{\varepsilon}-w_{\varepsilon}\right)=0, \text { in } \Omega \backslash \Omega_{\varepsilon} \\
\left(u_{\varepsilon}-w_{\varepsilon}\right)_{\Gamma_{a}}=0 \\
\left(u_{\varepsilon}-w_{\varepsilon}\right)_{\Gamma_{\varepsilon}}<0 .
\end{array}\right.
$$

From 27, and using the maximum principle, we can also conclude that $u_{\varepsilon}-w_{\varepsilon} \leq 0$, in $\overline{\Omega \backslash \Omega_{\varepsilon}}$ and, in fact, $u_{\varepsilon}-w_{\varepsilon}<0$, in $\Omega \backslash \Omega_{\varepsilon}$. As a consequence, using the definition of directional derivative, we find that $\left.\frac{\partial u_{\varepsilon}}{\partial n}\right|_{\Gamma_{a}} \geq\left.\frac{\partial w_{\varepsilon}}{\partial n}\right|_{\Gamma_{a}}$.

From $\left.\frac{\partial\left(u_{\varepsilon}-w_{\varepsilon}\right)}{\partial n}\right|_{\Gamma_{a}} \geq 0$ and $\int_{\Gamma_{a}} \frac{\partial\left(u_{\varepsilon}-w_{\varepsilon}\right)}{\partial n}=0$ we conclude that $\left.\frac{\partial\left(u_{\varepsilon}-w_{\varepsilon}\right)}{\partial n}\right|_{\Gamma_{a}}=0$. Therefore, we have $u_{\varepsilon}-w_{\varepsilon}<0$, in $\Omega \backslash \Omega_{\varepsilon}$, and $\left(u_{\varepsilon}-w_{\varepsilon}\right)=0$, in $\Gamma_{a}$. Using Lemma 3.4 of [2], for each point of $\Gamma_{a}$, we find $\frac{\partial\left(u_{\varepsilon}-w_{\varepsilon}\right)}{\partial n}>0$ a.e. on $\Gamma_{a}$ and we reach a contradiction. So, we must have $\inf _{\Gamma_{\varepsilon}} w_{\varepsilon} \leq c_{\varepsilon}$.

Analogously, one can show that $c_{\varepsilon} \leq \sup _{\Gamma_{\varepsilon}} w_{\varepsilon}$.

For the second part of the proof, using [2] (Theorem 8.15, page 189, with $q=4$ ), we can show that $\left\|w_{\varepsilon}\right\|_{L^{\infty}(\Omega)}$ is bounded by a constant not depending on $\varepsilon$ (it only depends on constants concerning $\|f\|_{L^{2}(\Omega)}$ and the size of $\Omega$ ) and the result follows.

Now we are able to establish the value of $u$ on the origin. 
Theorem 3. Let $f \in \mathscr{C}^{0, \alpha}(\Omega)$ Then, when $\varepsilon$ converges to $0, u_{\varepsilon_{\left.\right|_{\varepsilon}}}$ converges to $u(0)$.

Proof. Considering $u$ the solution of problem $(\mathscr{P})$, since $f \in \mathscr{C}^{0, \alpha}(\Omega)$, we have $u \in$ $\mathscr{C}^{2, \alpha}(\Omega)$. Let, as previously, $-\Delta w_{\varepsilon}=\tilde{f}_{\varepsilon}, w_{\varepsilon} \in H_{0}^{1}(\Omega)$. Therefore, $v_{\varepsilon}=w_{\varepsilon}-u$ satisfies $-\Delta\left(v_{\varepsilon}\right)=\tilde{g_{\varepsilon}}$, where $\tilde{g_{\varepsilon}}=\left\{\begin{array}{c}-f, \Omega_{\varepsilon} \\ 0, \Omega \backslash \Omega_{\varepsilon} \text {. }\end{array}\right.$ Using again [2] we can show that $\left\|v_{\varepsilon}\right\|_{L^{\infty}(\Omega)} \leq k\left(\left\|v_{\varepsilon}\right\|_{L^{2}(\Omega)}+\left\|\tilde{g_{\varepsilon}}\right\|_{L^{2}(\Omega)}\right)$, where $k$ is a constant not depending on $\varepsilon$. When $\varepsilon \rightarrow 0$ we have $\left\|v_{\varepsilon}\right\|_{L^{2}(\Omega)} \rightarrow 0$ and $\left\|\tilde{g_{\varepsilon}}\right\|_{L^{2}(\Omega)} \rightarrow 0$, then $\left\|v_{\varepsilon}\right\|_{L^{\infty}(\Omega)} \rightarrow 0$. So, for $\delta>0$ there exists $\varepsilon>0$ such that $\left|v_{\varepsilon}(x)\right| \leq \frac{\delta}{2}$ and $|u(x)-u(0)| \leq \frac{\delta}{2}, \forall x \in \Omega_{\varepsilon} \cup \Gamma_{\varepsilon}$. Then, for $x \in \Gamma_{\varepsilon},\left|w_{\varepsilon}(x)-u(0)\right|=\left|v_{\varepsilon}(x)+u(x)-u(0)\right| \leq \delta$ and consequently, $-\delta \leq \inf _{\Gamma_{\varepsilon}}\left(w_{\varepsilon}(x)\right)$ $-u(0)=\inf _{\Gamma_{\varepsilon}}\left(w_{\varepsilon}(x)-u(0)\right) \leq \sup _{\Gamma_{\varepsilon}}\left(w_{\varepsilon}(x)-u(0)\right)=\sup _{\Gamma_{\varepsilon}}\left(w_{\varepsilon}(x)\right)-u(0) \leq \delta$. Using (23), we find $-\delta \leq \inf _{\Gamma_{\varepsilon}} w_{\varepsilon}-u(0) \leq\left. u_{\varepsilon}\right|_{\Gamma_{\varepsilon}}-u(0) \leq \sup _{\Gamma_{\varepsilon}} w_{\varepsilon}-u(0) \leq \delta$, which implies that $\left.u_{\varepsilon}\right|_{\Gamma_{\varepsilon}} \rightarrow u(0)$, when $\varepsilon \rightarrow 0$.

\section{Conclusion}

Considering $H_{\tau, p}^{1}(\mathscr{I})$, where $\mathscr{I}$ denotes the interval $\left(0, t_{0}\right)$, to be the space of functions $v$ verifying $v \in L^{2}(\mathscr{I}), \frac{1}{\cos (\alpha)} \frac{\partial v}{\partial t} \in L^{2}(\mathscr{I})$ and such that $v$ has periodic boundary conditions $v(0)=v\left(t_{0}\right)$, we can define $H_{\tau, p}^{1 / 2}(\mathscr{I})$ as the $1 / 2$ interpolate between $H_{\tau, p}^{1}(\mathscr{I})$ and $L^{2}(\mathscr{I})$, and $\left(H_{\tau, p}^{1 / 2}(\mathscr{I})\right)^{\prime}$ as the $1 / 2$ interpolate between $\left(H_{\tau, p}^{1}(\mathscr{I})\right)^{\prime}$ and $L^{2}(\mathscr{I})$. The final result is synthesized as follows - denoting by $(.,$.$) the scalar product in L^{2}(\mathscr{I})$, then $P, r$ and $u_{\tau}$ satisfy:

1. The operator

$$
\begin{aligned}
P \in \mathscr{L}\left(L^{2}(\mathscr{I}), H_{\tau, p}^{1}(\mathscr{I})\right) & \cap \mathscr{L}\left(\left(H_{\tau, p}^{1 / 2}(\mathscr{I})\right)^{\prime}, H_{\tau, p}^{1 / 2}(\mathscr{I})\right) \\
& \cap \mathscr{L}\left(\left(H_{\tau, p}^{1}(\mathscr{I})\right)^{\prime}, L^{2}(\mathscr{I})\right),
\end{aligned}
$$

bounded as a function of $\tau$, satisfies, for every $h, \bar{h}$ in $L^{2}(\mathscr{I})$, the Riccati equation

$$
\begin{aligned}
& \left(\frac{d P}{d \tau} h, \bar{h}\right)-\left(\frac{\varphi \sin \alpha}{\tau} h, \frac{\partial}{\partial t} \circ P \bar{h}\right)-\left(\frac{\partial}{\partial t} \circ P h, \frac{\varphi \sin \alpha}{\tau} \bar{h}\right) \\
& -\left(\frac{\varphi \cos \alpha}{\tau^{2}} \frac{\partial}{\partial t} \circ P h, \frac{\partial}{\partial t} \circ P \bar{h}\right)-\left(\frac{1}{\tau} h, P \bar{h}\right)=-(\varphi \cos \alpha h, \bar{h})
\end{aligned}
$$

in $\mathscr{D}^{\prime}(0,1)$, with the initial condition $P(1)=0$.

2. For every $h$ in $H_{\tau, p}^{1 / 2}(\mathscr{I}), r$ satisfies the equation

$$
\left(\frac{\partial r}{\partial \tau}, h\right)-\left(\frac{\varphi \sin \alpha}{\tau} \frac{\partial r}{\partial t}, h\right)-\left(\frac{\varphi \cos \alpha}{\tau^{2}} \frac{\partial r}{\partial t}, \frac{\partial}{\partial t} \circ P h\right)=-(\varphi \cos \alpha f, P h)
$$

in $\mathscr{D}^{\prime}(0,1)$, with the initial condition $r(1)=0$. 
3. For every $h$ in $\left(H_{\tau, p}^{1 / 2}(\mathscr{I})\right)^{\prime}, u$ satisfies the equation

$$
\begin{aligned}
\left(\frac{1}{\varphi \cos \alpha} \frac{\partial u}{\partial \tau}, P h\right)-\left(\frac{\tan \alpha}{\tau} \frac{\partial u}{\partial t}, P h\right) & +\langle u, h\rangle_{H_{\tau, p}^{1 / 2}(\mathscr{I}),\left(H_{\tau, p}^{1 / 2}(\mathscr{I})\right)^{\prime}} \\
& =\langle r, h\rangle_{H_{\tau, p}^{1 / 2}(\mathscr{I}),\left(H_{\tau, p}^{1 / 2}(\mathscr{I})\right)^{\prime}}
\end{aligned}
$$

in $\mathscr{D}^{\prime}(0,1)$, with the initial condition given by Theorem 3

Moreover, taking $Q=\frac{P}{\tau}$ as unknown and $\mu=\log (\tau)$ as variable, the above Riccati equation can also take the simpler form

$$
\begin{aligned}
& \left(\frac{d Q}{d \mu} h, \bar{h}\right)-\left(\varphi \sin \alpha h, \frac{\partial}{\partial t} \circ Q \bar{h}\right)-\left(\frac{\partial}{\partial t} \circ Q h, \varphi \sin \alpha \bar{h}\right) \\
& -\left(\varphi \cos \alpha \frac{\partial}{\partial t} \circ Q h, \frac{\partial}{\partial t} \circ Q \bar{h}\right)=-(\varphi \cos \alpha h, \bar{h}),
\end{aligned}
$$

and, identically, the equation for the residue $r$ becomes

$$
\left(\frac{\partial r}{\partial \mu}, h\right)-\left(\varphi \sin \alpha \frac{\partial r}{\partial t}, h\right)-\left(\varphi \cos \alpha \frac{\partial r}{\partial t}, \frac{\partial}{\partial t} \circ Q h\right)=-\left(e^{2 \mu} \varphi \cos \alpha f, Q h\right),
$$

with initial conditions, respectively, $Q(0)=0$ and $r(0)=0$. Then $u$ satisfies

$$
\begin{aligned}
& \left(\frac{1}{\varphi \cos \alpha} \frac{\partial u}{\partial \mu}, Q h\right)-\left(\tan \alpha \frac{\partial u}{\partial t}, Q h\right)+\langle u, h\rangle_{H_{\tau, p}^{1 / 2}(\mathscr{I}),\left(H_{\tau, p}^{1 / 2}(\mathscr{I})\right)^{\prime}} \\
& =\langle r, h\rangle_{H_{\tau, p}^{1 / 2}(\mathscr{I}),\left(H_{\tau, p}^{1 / 2}(\mathscr{I})\right)^{\prime}}
\end{aligned}
$$

The initial condition on $u$ given by Theorem 3 is now valid at $\mu=-\infty$. These equations allow us to seek an explicit formula for the solution of $(\mathscr{P})$, through homographic transformation, as the Riccati equation has constant coefficient in $\mu$.

From the numerical point of view, one can consider a spatial discretization of the problem adapted to the system of coordinates $(t, \tau)$ (or $(t, \mu)$ ), which leads to a linear system of equations. Then there exists a particular discretization of the system (32), (33), (34) through which we can recover the Gauss block $L U$ factorization of this linear system. That is why we claim that the proposed factorization is an infinite dimensional generalization of the Gauss factorization. But other discretizations exist that give new directly computable discretizations of the original Poisson boundary value problem. They will be presented elsewhere.

\section{References}

1. Bellman, R.: Dynamic Programming. Princeton University Press, Princeton (1957)

2. Gilbarg, D., Trudinger, N.: Elliptic Partial Differential Equations of Second Order. Springer, Berlin (1983)

3. Henry, J., Louro, B., Soares, M.C.: A factorization method for elliptic problems in a circular domain. C. R. Math. Acad. Sci. 339(3), 175-180 (2004) 
4. Henry, J., Ramos, A.: Factorization of second order elliptic boundary value problems by dynamic programming. Nonlinear Analysis 59, 629-647 (2004)

5. Henry, J., Ramos, A.: Study of the initial value problems appearing in a method of factorization of second-order elliptic boundary value problems. Nonlinear Analysis 68, 2984-3008 (2008)

6. Lions, J.L.: Optimal Control of Systems Governed by Partial Differential Equations. Springer, New York (1971)

7. Soares, M.C.: Factorization by Invariant Embedding of Elliptic Problems: Circular and Starshaped Domains, PhD Thesis, Faculdade de Ciências e Tecnologia, Universidade Nova de Lisboa (2006) 